\title{
Treating Irritable Bowel Syndrome: A Fresh and Minty Approach to an Old Therapy
}

\author{
Lucinda A. Harris ${ }^{1}$
}

Published online: 21 December 2015

(c) Springer Science+Business Media New York 2015

At the hairdresser's the other day, the beautician applied a conditioner that contained peppermint oil (PO). My scalp felt cooled and soothed, and the scent was very calming. As I contemplated writing this editorial, it occurred to me that even my hairdresser was aware of the wisdom of the ages that PO is used to soothe and calm. In fact, the Internet touts 21 uses for PO, five of which are for digestive issues, with the number one indication being the treatment for irritable bowel syndrome (IBS).

IBS is defined as a chronic disorder of altered bowel function characterized by symptoms of diarrhea, constipation, or alternating bowel habits accompanied by pain or discomfort and may include a constellation of other symptoms, e.g., bloating, urgency, and incomplete evacuation. The pathophysiology of this disorder has been attributed to gut inflammation, altered visceral sensitivity, food sensitivities, gene susceptibility, and changes in the gut-host microbiome, and altered brain-gut signaling [1, 2]. The heterogeneity of this disorder has long presented challenges in developing treatments since it is unlikely that one intervention will treat all causes and relieve all symptoms. That being said, the characteristics of PO make it an interesting choice as a therapeutic agent.

The active ingredient of $\mathrm{PO}$ is the cyclic terpene L-menthol. The compound has anti-inflammatory, antioxidant, and anti-apoptotic activity as reported in animal studies in which PO was stated to heal gastric ulcers induced by either $1 \mathrm{ml} 96 \%$ ethanol or indomethacin

Lucinda A. Harris

Harris.Lucinda@mayo.edu

1 Division of Gastroenterology and Hepatology, Department of Medicine, Mayo College of Medicine, 13400 E. Shea Blvd., Scottsdale, AZ 85259, USA
$(100 \mathrm{mg} / \mathrm{kg})$ [3, 4], doses higher than typically ingested by humans. The reduction in average ulcer area produced corresponded to a gastroprotective effect for PO of $92 \%$ for alcohol and $73 \%$ for indomethacin. In these rat studies, L-menthol (50 mg/kg, lowest effective dose), a dose higher than in clinical use, induced mucus secretion maintained the production of prostaglandin $\mathrm{E}_{2}$, decreased concentrations of pro-inflammatory cytokines such as tumor necrosis factor (TNF)- $\alpha$ and interleukin (IL)-6, and increased concentrations of the anti-inflammatory cytokine interleukin10. Additionally, the antispasmodic activity of PO is thought to be due to inhibition of smooth muscle voltagegated $\mathrm{Ca}^{2+}$ channels $[5,6]$. Carrying this research one step further, a recent study has also reported the L-menthol can also inhibit 5- $\mathrm{HT}_{3}$ receptors in vitro, which may explain the decrease in the frequency and amplitude of gut contractions associated with PO [7].

This laboratory research notwithstanding, the clinical effects of PO have been mixed. The authors of a recent meta-analysis concluded that PO was safe and effective for the short-term treatment for the treatment of IBS [8]. Using the Cochrane bias tool, they identified nine studies involving 726 patients where the risk of bias was low for most of the factors assessed. PO was significantly superior to placebo for global relief of IBS symptoms in five studies $(N=392$ patients, RR 2.23, $95 \%$ confidence interval 1.78-2.81) and for improving abdominal pain in five studies $(N=357$ patients, RR $2.14,95 \%$ confidence interval 1.64-2.79). Even the recent American College of Gastroenterology guidelines, which gave PO a weak recommendation, reported that the level of evidence was moderate and stated, "Peppermint oil is superior to placebo in improving IBS symptoms. The risk of adverse events is no greater with PO than with placebo" [9]. The clinical trials on which these recommendations were based were 
not all randomized and controlled double-blind trials; some were simply crossover studies. Some compared PO to placebo, others to anticholinergic agents. Also, preparations varied in tolerability.

In this month's issue of Digestive Diseases and Sciences, Cash et al. published a small but noteworthy 4-week, double-blind, randomized, placebo-controlled clinical trial of a novel PO formulation (IBGard ${ }^{\circledR}$ ) in 72 subjects (18-60 y/o) meeting Rome III criteria for IBS (mixed or diarrhea predominant with an average pain of $\geq 4(0-10)$ ) with moderate to severe disease (Total IBS Symptom Score (TISS) $\geq 2(0-4)$ ) [10]. Eight symptoms were assessed: abdominal pain or discomfort, bloating or distention, pain at evacuation, urgency, constipation, and diarrhea, passage of mucus or gas, and sense of incomplete evacuation. The TISS was calculated by adding the means of intensity and frequency for each assessed IBS symptom and dividing by 8. The frequency and symptom intensity were both on a scale of 0 (no symptoms) to 4 (very severe and $\geq 3$ times/ week).

The primary end point was the change in baseline in the TISS after 4 weeks of treatment. Not only were global scores significantly improved at $24 \mathrm{~h}$ (19.6 vs. $10.3 \%)$ and at 4 weeks (40 vs. $24.3 \%$ ) but additionally $4 / 8$ secondary end points (abdominal pain, bloating/distention, pain at evacuation and urgency) were also significantly improved.

Six subjects reported that of a total of six treatmentemergent adverse events (TEAEs), four occurred in the placebo group compared with two in the PO group. All events were mild with the exception of moderate GERD in one patient in the placebo group. Of note, none of the patients in the PO group reported smelling menthol in the breath, stool, or flatus, and none reported anal discomfort.

Although the design of this trial was carefully thought out, there may still be some shortcomings when compared to the more rigorously designed trials needed for Food and Drug Administration (FDA) approval. First, this is a small study not comparable to the large trials used to test other IBS drugs such as linaclotide and lubiprostone in which 800-1600 patients were enrolled. The length of the trial is also short-4 weeks-when compared to the 12- to 26-week observation period used for other medications. Interestingly, PO study subjects were excluded from taking anticholinergic medications, probiotics, insoluble fiber, antidepressants, anxiolytics, opioids, nonsteroidal anti-inflammatory drugs (NSAIDs), antispasmodics, and antibiotics (except for topical agents or a 1-day course) potentially skewing the population to a group somewhat different from that studied by other clinical trials where stable doses of antidepressants or sometimes other drugs such as NSAIDs are allowed. It may also mean this patient group had less severe concomitant psychopathology since antidepressants and anxiolytics were not allowed.
Another area of concern is that the TISS scale used by the authors is not a validated scale but was chosen because it had been used in another PO trial [11]. The FDA and the European Medicines Agency (EMA) have discouraged the use of global scales, citing that they do not adequately assess the degree of improvement or worsening of symptoms nor do they provide information about the improvement of individual symptoms [12, 13]. The most recent FDA directives favor a composite end point of assessing relief of abdominal pain and bowel function, i.e., change in consistency/frequency of stool [12]. These two symptoms were chosen because they were considered the most bothersome in IBS patients. Nevertheless, the controversy surrounding proper end points is ongoing. Camilleri et al. [14], in a recent analysis of the linaclotide data, argue that a simple criterion, adequate relief of symptoms, rather than global end points, abdominal symptoms, or composite symptoms, equally assesses clinically meaningful change and would also enable a better comparison of trial results. One might argue that this study did indirectly measure clinically meaningful change because the four symptoms that improved (abdominal pain/discomfort, abdominal bloating, pain at evacuation, and urgency) reflect symptoms of "viscerosensory perception" in IBS patients that the number and consistency of bowel movements do not reflect.

Although the best means of assessing response to IBS medications can be argued, one point that is striking is that the PO product used by Cash et al. is an innovative patented targeted delivery system (Site-Specific Targeting $\left(\mathrm{SST}^{\circledR}\right)$ technology) that embeds the active component in a matrix that stays intact until it reaches the small intestine, which is thought to be an important site of primary IBS symptoms [15]. Other formulations have been associated with adverse effects such as nausea, heartburn, and anal burning as well as with inconsistent breakdown [16, 17]. Whether this time release formulation is significantly different than other enteric-coated PO (e.g., Colpermin ${ }^{\circledR}$ ) on the market will remain to be proven by clinical use.

Another noteworthy item is that the compound being evaluated here is classified as a "medical food" and as such highlights this entire therapeutic area. A medical food is defined as "a food which is formulated to be consumed or administered enterally under the supervision of a physician and which is intended for the specific dietary management of a disease or condition for which distinctive nutritional requirements, based on recognized scientific principles, are established by medical evaluation" [18]. It is different than other drugs as it is not subject to approval by the FDA, but rather that the food facility producing it is registered and subject to "Good Manufacturing Practices" under FDA regulatory statutes. There are special labeling requirements such as that the product must contain ingredients that are 
Generally Recognized as Safe (GRAS), meaning no added sugar, salt, fat, gluten, wheat, starch, yeast, corn, lactose, egg, soy, peanuts, shellfish, or preservatives. This is the second compound to be recently registered for the treatment of IBS-D, the other being the bovine serum isolate $\left(\right.$ EnteraGam $\left.^{\circledR}\right)$ [19].

Although this PO preparation appears to be promising for the treatment of IBS, it does not supplant what are considered the most effective comprehensive therapeutic tools for IBS such as symptom validation, empathy, and optimal clinician-patient interactive skills along with sound dietary counseling and the achievement of better sleep quality and more exercise, combined at times with cognitive behavioral therapy. Given the growing body of information on the microbiome and its contributions to the pathophysiology of IBS, PO with its anti-bacterial effects may offer beneficial anti-nociceptive and anti-inflammatory effects, certainly more soothing than yet another diagnostic test $[20,21]$.

\section{References}

1. Camilleri M. Peripheral mechanisms in irritable bowel syndrome. New Engl J Med. 2012;367:1626-1635.

2. Ohman L, Simren M. Pathogenesis of IBS: role of inflammation, immunity and neuroimmune interactions. Nat Rev Gastroenterol Hepatol. 2010;7:163-173.

3. Rozza AL, Hiruma-Lima CA, Takahira RK, Padovani CR, Pellizon $\mathrm{CH}$. Effect of menthol in experimentally induced ulcers: pathways of gastroprotection. Chem Biol Interact. 2013;206: 272-278.

4. Rozza AL, Meira de Faria F, Souza Brito AR, Pellizzon CH. The gastroprotective effect of menthol: involvement of anti-apoptotic, antioxidant and anti-inflammatory activities. Plos One. 2014;9:1-6.

5. Hills JM, Aaronson PI. The mechanism of action of peppermint oil on gastrointestinal smooth muscle: an analysis using patch clamp electro-physiology and isolated tissue pharmacology in rabbit and guinea pig. Gastroenterology. 1991;101:55-65.

6. Hawthorn M, Ferrante J, Luchowski E, Rutledge A, Wei XY, Triggle DJ. The actions of peppermint oil and menthol on calcium channel dependent processes in intestinal, neuronal and cardiac preparations. Aliment Pharmacol Ther. 1988;2:101-118.

7. Walstab J, Wohlfarth C, Hovius R, et al. Natural compounds boldine and menthol are antagonists of human 5-HT3 receptors: implications for treating gastrointestinal disorders. Neurogastroentorol Motil. 2014;26:810-820.
8. Khanna R, MacDonald J, Levesque B. Peppermint oil for the treatment of irritable bowel syndrome: a systematic review and meta-analysis. J Clin Gastroenterol. 2014;48:505-512.

9. Ford AC, Moayeddi P, Lacy B, et al. American college of gastroenterology monograph on the management of irritable bowel syndrome and chronic idiopathic constipation. Am J Gastroenterol. 2014;109:S2-S26.

10. Cash BD, Epstein MS, Shah SM. A novel delivery system of peppermint oil is an effective therapy for irritable bowel syndrome symptoms. Dig Dis Sci (Epub ahead of print). doi:10.1007/ s10620-015-3858-7.

11. Cappello G, Spezzaferro M, Grossi L, Manzoli L, Marzio L. Peppermint oil (Mintoil) in the treatment of irritable bowel syndrome: a prospective double blind placebo-controlled randomized trial. Dig Liver Dis. 2007;39:530-536.

12. U.S. Department of Health and Human Services Food and Drug Administration Center for Drug Evaluation and Research (CDER). Guidance for Industry: Irritable Bowel SyndromeClinical Evaluation of Drugs for Treatment; 2012. Available at: http://www.fda.gov/downloads/Drugs/Guidances/UCM205269. pdf.

13. Committee for Medicinal Products for Human Use. Guideline on the Evaluation of Medicinal Products for the Treatment of Irritable Bowel Syndrome. CPMP/EWP/785/97 2014; Rev. 1:1-18. Available at: http://www.ema.europa.eu/docs/en_GB/document_ library/Scientific_guideline/2013/07/WC500146176.pdf.

14. Camilleri M, Lembo AJ, Lavins BJ, et al. Comparison of adequate relief with symptom, global, and responder endpoints in linaclotide phase 3 trials in IBS-C. United Eur Gastroenterol J. 2015;3:53-62.

15. http://ibgard.com/overview.aspx. U.S. Patent No.: 8,808,736.

16. Grigleit HG, Grigleit P. Peppermint oil in irritable bowel syndrome. Phytomedicine. 2005;12:601-606.

17. Somerville KW, Richmond CR, Bell GD. Delayed release peppermint oil capsules (Colpermin) for the spastic colon syndrome: a pharmacokinetic study. Br J Clin Pharmacol. 1984;18:638-640.

18. US Food and Drug Administration. Medical Foods Documents and Regulatory Information. Available at: http://www.fda.gov/ Food/GuidanceRegulation/GuidanceDocumentsRegulatoryInfor mation/MedicalFoods/. Accessed September 17, 2015.

19. Wilson D, Evans M, Weaver E, Shaw AL, Klein GL. Evaluation of a serum-derived bovine immunoglobulin protein isolate in subjects with diarrhea-predominant irritable bowel syndrome. Clin Med Insights Gastroenterol. 2013;6:49-60.

20. Imai H, Osawa K, Yasuda H, Hamashima H, Arai T, Sasatsu M. Inhibition by the essential oils of peppermint and spearmint of the growth of pathogenic bacteria. Microbios. 2001;106:31-39.

21. O'Mahony L, McCarthy J, Kelly P, et al. Lactobacillus and bifidobacterium in irritable bowel syndrome: symptom responses and relationship to cytokine profiles. Gastroenterology. 2005; 128:541-551. 